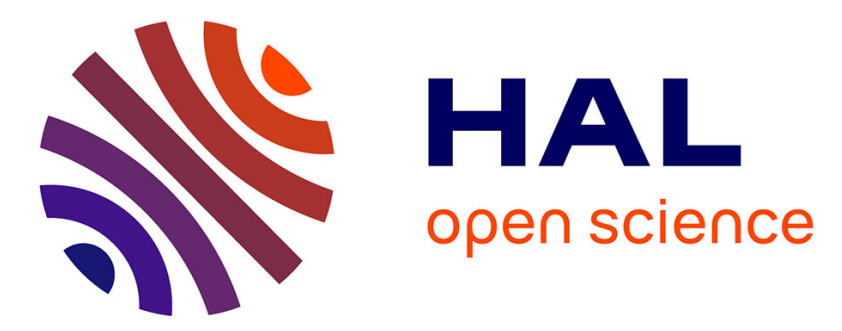

\title{
A Mortar BDD method for solving flow in stochastic discrete fracture networks
}

Géraldine Pichot, Baptiste Poirriez, Jocelyne Erhel, Jean-Raynald de Dreuzy

\section{To cite this version:}

Géraldine Pichot, Baptiste Poirriez, Jocelyne Erhel, Jean-Raynald de Dreuzy. A Mortar BDD method for solving flow in stochastic discrete fracture networks. Jocelyne Erhel and Martin Gander and Laurence Halpern and Géraldine Pichot and Taoufik Sassi. Domain Decomposition Methods in Science and Engineering XXI, 98, Springer, 2014, Lecture Notes in Computational Science and Engineering (LNCSE). hal-00853248

\section{HAL Id: hal-00853248 \\ https://hal.inria.fr/hal-00853248}

Submitted on 9 Oct 2013

HAL is a multi-disciplinary open access archive for the deposit and dissemination of scientific research documents, whether they are published or not. The documents may come from teaching and research institutions in France or abroad, or from public or private research centers.
L'archive ouverte pluridisciplinaire HAL, est destinée au dépôt et à la diffusion de documents scientifiques de niveau recherche, publiés ou non, émanant des établissements d'enseignement et de recherche français ou étrangers, des laboratoires publics ou privés. 


\title{
A Mortar BDD method for solving flow in stochastic discrete fracture networks
}

\author{
Géraldine Pichot ${ }^{1}$, Baptiste Poirriez ${ }^{2}$, Jocelyne Erhel ${ }^{1}$, and Jean-Raynald de \\ Dreuzy $^{3}$ \\ 1 Introduction
}

In geological media, the large variety and complex configurations of fractured networks make it difficult to describe them precisely. A relevant approach is to model them as Discrete Fracture Networks (DFN)[10, 19], with statistical properties in agreement with in situ experiments $[15,13,14]$. A DFN is a 3D domain made of 2D fractures intersecting each other. Steady state flow in DFN is considered, the rock matrix is assumed impervious. Following a Monte-Carlo approach, a large number of DFN has to be generated and for each, a flow problem has to be solved whatever the complexity of the generated networks. Moreover time and memory costs for each simulation should be as lower as possible.

A nonconforming discretization of DFN allows to reduce the number of unknowns and facilitate mesh refinement. Sharp angles are managed by a staircaselike discretizations of the fractures' contours [34]. The non-matching feature at the fractures' intersections is handled via a Mortar method $[4,5,1]$ developed for DFN in $[33,34]$ for a mixed hybrid finite element formulation. It consists in defining, for each intersection between fractures, master and slave sides. Due to the staircaselike discretizations, a shared edge may be labeled several times with master and/or slave properties, it is called in the paper a multi-labeled edge. Continuity conditions are enforced between the unknowns on both sides. The derived linear system has only inner and master traces of hydraulic head as unknowns. The matrix $A$ of this system is a symmetric definite positive (SPD) arrow matrix in presence of Dirichlet boundary conditions [34].

The challenge is to solve such linear systems with millions of unknowns [17]. Direct solvers (like Cholmod [11]) are very efficient for small systems but suffer from a high need of RAM memory when the system size becomes too large. Among iterative solvers, multigrid methods are very efficient for most networks but for some, the convergence rate is very slow [35, 17]. Preconditioned Conjugate Gradient (PCG) is efficient and robust for every network tested [35]. The natural decomposition of the matrix $A$ in subdomains encourages the use of domain decomposition methods $[7,36,31,24]$. The Schur complement of the matrix $A$ is SPD and yields an interface system with only master unknowns. This interface system can be solved iteratively

1 INRIA Rennes Bretagne-Atlantique, Campus de Beaulieu, 35042 Rennes Cedex, email: \{geraldine.pichot\} jocelyne.erhel\}@inria.fr .2 IRISA, Campus de Beaulieu, 35042 Rennes Cedex, e-mail: baptiste.poirriez@irisa.fr ${ }^{3}$ Géosciences Rennes, Campus de Beaulieu, 35042 Rennes Cedex, e-mail: Jean-Raynald.de-Dreuzye univ-rennes1.fr 
with PCG. The unknowns on inner edges are then derived locally in each fracture plane by solving small local linear systems, with a direct solver for example.

Among possible preconditioners, the balancing domain decomposition (BDD) method is based on a Neumann-Neumann preconditioner coupled with a coarse level solver, to improve the preconditioner as the number of subdomains increases [29, 30, 27]. BDD method applied to mixed finite element is done in [12]. The application to a nonconforming discretization is proposed in [18, 32]. Meanwhile, an alternative method has been developed, the Balancing Domain Decomposition by Constraints (BDDC) [16], later applied to mortar discretization for geometrically nonconforming partitions in [26].

In this paper, we use the BDD algorithm proposed in $[32,35]$ to solve the linear system arising from a nonconforming discretization of DFN. The coarse level is defined following [37] and balancing is implemented as a preconditioning matrix [21]. The algorithm is implemented in C++ in the parallel software SIDNUR [35]. For DFN, choosing one subdomain given by one fracture, instead of a set of fractures has shown to be the most time saving decomposition [35].

The paper is organized in four sections. Section 2 describes the flow model. Section 3 recalls the linear system derived from a nonconforming discretization of the DFN. Section 4 is the main contribution of this paper and presents the decomposition in local matrices. We apply the BDD method proposed in $[32,35]$ for networks satisfying some hypotheses on the mesh. The last section illustrates the application of the solver SIDNUR [35] on three stochastically generated DFN.

\section{Flow model}

We consider flow in DFN assuming the rock matrix is impervious. In the entire paper, an intersection is uniquely defined as the segment shared by two fractures. We denote $\Sigma_{k}$ the kth intersection, $k=1, \ldots, N_{i}$.

Poiseuille's law and mass conservation apply in each fracture plane, denoted $\Omega_{f}$, $f=1, \ldots, N_{f}$. We assume there is no longitudinal flux at the fracture intersections.

The DFN is embedded in a cube of size $L$. Some fractures are truncated by the cube faces. Classical permeameter boundary conditions apply on the cube faces. The two opposite faces of the cube with Dirichlet boundary conditions (prescribed value $\left.p^{D}\right)$ are called $\Gamma_{D}\left(\Gamma_{D} \neq \emptyset\right)$ and the lateral faces with homogeneous Neumann boundary conditions are called $\Gamma_{N}$. The boundary of the fracture $f$ is called $\Gamma_{f}$. In the following, we assume there is only one cluster of fractures connected to the Dirichlet boundary conditions and we consider only this cluster.

In each fracture plane, with $x \in \mathbb{R}^{2}$, the following equations link the unknown hydraulic head scalar function $p(x)$ and the flux per unit length function $u(x)$ :

$$
\begin{aligned}
\nabla \cdot u(x)=f(x) & \text { for } x \in \Omega_{f}, \\
u(x)=-\mathscr{T}(x) \nabla p(x) & \text { for } x \in \Omega_{f}, \\
p(x)=p^{D}(x) & \text { on } \Gamma_{D} \cap \Gamma_{f},
\end{aligned}
$$




$$
\begin{array}{ll}
u(x) \cdot v=0 & \text { on } \Gamma_{N} \cap \Gamma_{f}, \\
u(x) \cdot \mu=0 & \text { on } \Gamma_{f} \backslash\left\{\left(\Gamma_{f} \cap \Gamma_{D}\right) \cup\left(\Gamma_{f} \cap \Gamma_{N}\right)\right\},
\end{array}
$$

where $v$ (respectively $\mu$ ) denotes the outward normal unit vector of the borders with respect to the fracture $\Omega_{f}$. The parameter $\mathscr{T}(x)$ is a given SPD transmissivity field (unit $\left[\mathrm{m}^{2} . \mathrm{s}^{-1}\right]$ ). The function $f(x) \in L^{2}\left(\Omega_{f}\right)$ represents the sources/sinks.

Let $\mathscr{I}_{l}$ be a segment shared by several incident fractures, $l=1, \ldots, N_{l}$. It can be the intersection itself or only a part of it if intersections overlap. Let $F_{l}$ be the set of fractures which contains $\mathscr{I}_{l}$. On each segment, continuity conditions are imposed to ensure the continuity of hydraulic heads and the conservation of fluxes [20], [38]:

$$
\begin{aligned}
p_{f, l}=p_{l} & \text { on } \mathscr{I}_{l}, \forall f \in F_{l}, \\
\sum_{f \in F_{l}} u_{f, l} \cdot n_{f, l}=0 & \text { on } \mathscr{I}_{l},
\end{aligned}
$$

where $p_{f, l}$ is the trace of hydraulic head on $\mathscr{I}_{l}$ in the fracture $\Omega_{f}, p_{k}$ is the unknown hydraulic head on the segment $\mathscr{I}_{l}$ and $u_{f, l} . n_{f, l}$ is the normal flux through $\mathscr{I}_{l}$ coming from the fracture $\Omega_{f}$, with $n_{f, l}$ the outward normal unit vector of the segment $\mathscr{I}_{l}$ with respect to the fracture $\Omega_{f}$.

\section{A Mortar method applied to DFN}

\subsection{Mesh generation}

With a stochastic generation, fractures can cross in a very intricate way. We define the contour of a fracture $f$ as its border and all segments $\mathscr{I}_{l}$ which belong to $f$. To preserve a good mesh quality whatever the generated fractured networks, staircase like discretizations of the contour are performed in each fracture plane.

Each fracture is meshed with its own mesh step:

1. A temporary uniform grid is built that encompasses the fracture, with a grid step chosen as input;

2. 1D staircase-like meshes of the contour are built using the centers of the grid elements as discretization points;

3. From these 1D discretizations, a 2D triangle mesh of the fracture is built.

We call shared edges the edges of the triangles that discretize the segments $\mathscr{I}_{l}$, $l=1, \ldots, N_{l}$ within the different fractures in $F_{l}$. All other edges are called inner edges. Notice a given segment $\mathscr{I}_{l}$ may have different discretizations in the different fractures in $F_{l}$ as shown on figure 1 . The total mesh is made of $N_{i n}$ inner edges and of $N_{\Sigma}$ shared edges. In the following, we will use the subscript in to refer to the inner edges and $\Sigma$ to shared edges. 

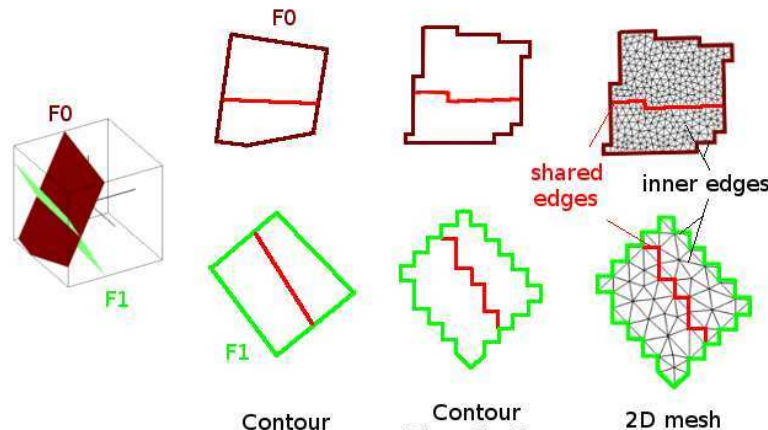

Contour

Contour
Discretization

2D mesh

Fig. 1 Mesh generation - Simple example with two fractures

\subsection{Derivation of the linear system}

The Mortar method applied to DFN is presented in [34]. It consists, for each intersection $\Sigma_{k}$, of choosing a master fracture $m$ and a slave fracture $s$. We denote $N_{m}=\sum_{k=1}^{N_{i}} N_{k, m}, N_{s}=\sum_{k=1}^{N_{i}} N_{k, s}$, with $N_{k,\{m, s\}}$ the number of edges that discretize the master (respectively slave) side of the intersection $\Sigma_{k}$.

The traces of hydraulic head unknowns are $\Lambda_{i n}$ on inner edges, $\Lambda_{m}$ and $\Lambda_{s}$ on master and slave edges. Additionnally, each shared edge has an unknown called $\Lambda_{\Sigma}$. The additional unknowns $\Lambda_{\Sigma}$ allow to deal with multi-labeled edges which belong to several intersections. The unknowns $\Lambda_{s}$ and $\Lambda_{\Sigma}$ are derived from $\Lambda_{m}$ following the relations (see [34]):

$$
\begin{array}{r}
\Lambda_{s}=C \Lambda_{m}, \\
\Lambda_{\Sigma}=P_{m} \Lambda_{m}+P_{S} \Lambda_{s}=\left(P_{m}+P_{s} C\right) \Lambda_{m} .
\end{array}
$$

The matrix $C$ is an intersection block matrix of dimension $N_{s} \mathrm{x} N_{m}$, with the block $C_{k}$ a matrix of size $N_{k, s} \mathrm{x} N_{k, m}$ for the intersection $\Sigma_{k}$ that represents the $L^{2}$-projection from the master side to the slave side.

Let denote $m_{E}$ (respectively $s_{E}$ ) the number of times a shared edge $E$ is labeled with a master (respectively slave) property. Let $n_{E}=s_{E}+m_{E}$. The values $(i, j)$ of the matrices $P_{m}$ (respectively $P_{s}$ ) of size $N_{\Sigma} \mathrm{x} N_{m}$ (respectively $N_{\Sigma} \mathrm{x} N_{s}$ ) is $\frac{1}{n_{E}}$ if the unknown $\Lambda_{m}(j)$ (respectively $\Lambda_{s}(j)$ ) is associated to an edge with $\Lambda_{\Sigma}(i)$ as shared unknown, and 0 otherwise.

At the network scale, the linear system reduces to a system with unknowns $\Lambda_{\text {in }}$ and $\Lambda_{m}$ [34]: 


$$
A\left(\begin{array}{c}
\Lambda_{i n} \\
\Lambda_{m}
\end{array}\right)=\left(\begin{array}{c}
F_{i n} \\
F_{m}
\end{array}\right)
$$

The second member is a vector of dimension $N_{i n}+N_{m}$, which corresponds to the source/sink function, to the imposed Dirichlet and Neumann boundary conditions.

The matrix $A$ is SPD in presence of Dirichlet boundary conditions [34] and writes as:

$$
\left\{\begin{aligned}
A & =\left(\begin{array}{ll}
A_{i n, i n} & A_{i n, m} \\
A_{i n, m}^{T} & A_{m, m}
\end{array}\right), \\
A_{i n, m} & =A_{i n, \Sigma}\left(P_{m}+P_{s} C\right), \\
A_{m, m} & =\left(P_{m}+P_{s} C\right)^{T} A_{\Sigma, \Sigma}\left(P_{m}+P_{S} C\right) .
\end{aligned}\right.
$$

The matrix $A_{\text {in }, \text { in }}$ is a block diagonal matrix of order $N_{\text {in }}$ made of blocks $A_{f, i n, i n}$ associated to the inner edges in the fracture $\Omega_{f}$.

\section{A Mortar BDD method for DFN system}

The arrow shape of the matrix $A$ allows to reduce the linear system (10) to an interface problem with only $\Lambda_{m}$ as unknowns:

$$
\begin{array}{r}
S \Lambda_{m}=B_{m}, \\
S=A_{m, m}-A_{i n, m}^{T} A_{i n, i n}^{-1} A_{i n, m}, \\
B_{m}=F_{m}-\left(P_{m}^{T}+C^{T} P_{s}^{T}\right) A_{i n, \Sigma}^{T} A_{i n, i n}^{-1} F_{i n} .
\end{array}
$$

with $S$ the Schur complement of size $N_{m} \mathrm{x} N_{m}$.

Since $S$ is SPD, the linear system (12) can be solved iteratively via a PCG method. To apply a balancing preconditioner, we need the local Schur complements $S_{f}, f=1, \ldots, N_{f}$.

\subsection{Local Schur complements}

Let $N_{f, m}$ (respectively $N_{f, s}$ ) be the number of master (respectively slave) unknowns associated with master (respectively slave) edges in the fracture $f$. Let $N_{f, o}$ be the number of master unknowns associated with the slave edges in the fracture $f$ following the relations (8). Let $N_{f, \Sigma}$ be the number of shared edges in the fracture $f$. We define the local matrices $\left(P_{m}+P_{s} C\right)_{f}$ as:

$$
\left(P_{m}+P_{s} C\right)_{f}=\left(P_{f, m} P_{f, s} C_{f}\right)
$$

with $P_{f, m}$ of size $N_{f, \Sigma} \mathrm{x} N_{f, m}$ and $P_{f, s}$ of size $N_{f, \Sigma} \mathrm{x} N_{f, s}$. The matrix $C_{f}$ of size $N_{f, s} \mathrm{x} N_{f, o}$ is a block matrix whose blocks $C_{k}$ are extracted from the matrix $C$ for the intersections $\Sigma_{k}$ in the fracture $f$. 
The local problem in the fracture $f$ writes as:

$$
A_{f, \Sigma}=\left(\begin{array}{ll}
A_{f, i n, i n} & A_{f, i n, \Sigma} \\
A_{f, i n, \Sigma}^{T} & A_{f, \Sigma, \Sigma}
\end{array}\right)
$$

Its associated Schur complement writes as: $S_{f, \Sigma}=A_{f, \Sigma, \Sigma}-A_{f, i n, \Sigma}^{T} A_{f, i n, i n}^{-1} A_{f, i n, \Sigma}$.

At the fracture scale, local matrices $A_{f}$, of order $\left(N_{f, i n}+N_{f, m}+N_{f, o}\right)$ are built from $A_{f, \Sigma}$ :

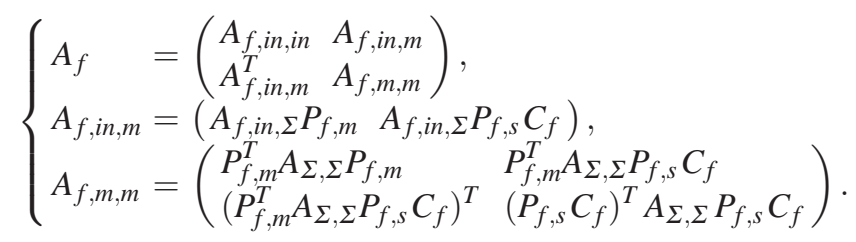

The block $A_{f, i n, m}$ is of size $N_{f, i n} \mathrm{x}\left(N_{f, m}+N_{f, o}\right)$ and the block $A_{f, m, m}$ is of size $\left(N_{f, m}+N_{f, o}\right) \mathrm{x}\left(N_{f, m}+N_{f, o}\right)$.

The local Schur complement $S_{f}$ associated to the matrix $A_{f}(17)$ of the fracture $\Omega_{f}$ writes:

$$
S_{f}=A_{f, m m}-A_{f, i n, m}^{T} A_{f, i n, i n}^{-1} A_{f, i n, m}=\left(P_{m}+P_{s} C\right)_{f}^{T} S_{f, \Sigma}\left(P_{m}+P_{s} C\right)_{f}
$$

As each intersection involves two fractures, one slave and one master, the Schur complement $S$ of size $N_{m} \mathrm{x} N_{m}$ is the sum of the local Schur complements:

$$
S=\sum_{f=1}^{N_{f}} R_{f}^{T} S_{f} R_{f}
$$

where $R_{f}$ is the rectriction matrix from the network to the fracture $f$.

\subsection{Neumann-Neumann preconditioner}

In the following, a subdomain $\Omega_{f}$ is said to be floating if it does not contain any Dirichlet boundary conditions, non floating otherwise.

The Neumann-Neumann preconditioner $[25,9,28]$ writes as:

$$
M_{N N}^{-1}=D \sum_{f} R_{f}^{T} S_{f}^{\dagger} R_{f} D
$$

where

$$
S_{f}^{\dagger}=\left\{\begin{array}{l}
S_{f}^{-1} \text { if } S_{f} \text { is non singular, } \\
\tilde{S}_{f}^{-1} \text { otherwise, with } \tilde{S}_{f} \text { a non singular approximation of } S_{f} .
\end{array}\right.
$$


The matrix $D$ is a diagonal matrix of order $N_{m}$. With a nonconforming discretization, a definition of one fracture as one subdomain and an homogeneous transmissivity, $D=\frac{1}{2} I d$ since each master unknown is defined for an intersection between two subdomains.

From the definition of $M_{N N}^{-1}$, one needs to solve local subdomain problems with the matrix $S_{f}$, like $S_{f} z_{f}=r_{f}$. However the kernel of $S_{f}$ may not be trivial. If the matrix $\left(P_{m}+P_{s} C\right)_{f}$ is of full rank, the kernel of $S_{f}$ is that if $S_{f, \Sigma}:\{0\}$ for a non floating subdomain, else $\{$ const $\}$. We assume that $\left(P_{m}+P_{s} C\right)_{f}$ is of full rank if the following conditions are satisfied:

(H1) the master side of an intersection must have the smallest number of discretization edges: $N_{k, m} \leq N_{k, s}, \forall k \in 1, \ldots, N_{i}$;

(H2) There are no multi-labeled edges: $n_{E}=1$ for each shared edge $E$ yielding: $N_{\Sigma}=N_{m}+N_{s}$.

If the subdomain is floating, in order to get a SPD approximation $\tilde{S}_{f}$, we add one arbitrary Dirichlet condition, since the kernel is of dimension 1 [35].

\subsection{Balancing preconditioner}

As the number of subdomains increases, the efficiency of the Neumann-Neumann preconditioner decreases [27] and one has to couple it with a coarse level solver $[29,30]$. We use the following balancing preconditioner:

$$
M_{b}^{-1}=P^{T} M_{N N}^{-1}
$$

as in $[37,21,35]$ where the projection matrix $P$, of order $N_{m}$, is defined as:

$$
P=I-S Z S_{c}^{-1} Z^{T}
$$

The matrix $Z$ is a $N_{m} \mathrm{x} N_{c}$ subspace matrix with full rank, $N_{c}<N_{m}$, and $S_{c}=$ $Z^{T} S Z$ is the invertible matrix corresponding to the coarse problem.

This formulation is based on the PCG initial value:

$$
\Lambda_{m, 0}=Z S_{c}^{-1} Z^{T} B_{m}
$$

such that, for all iterations it of PCG, the residuals $r_{i t}=S \Lambda_{m, i t}-B_{m}$ satisfy $Z^{T} r_{i t}=0$ and $P r_{i t}=r_{i t}$ [35]. Thus applying (22) is equivalent to apply $P^{T} M_{N N}^{-1} P+Z S_{c}^{-1} Z^{T}$ [37, 35].

A possible choice for the full rank matrix $Z$ is to use a subdomain deflation as defined in $[22,35]$. Here $N_{c} \leq N_{f}$ and $Z$ is sparse. 


\section{Numerical experiments}

We present preliminary numerical experiments on three random DFN that satisfy hypotheses $(H 1)-(H 2)$, generated with the software MP_FRAC of the H2OLab platform http://h2olab.inria.fr/. We checked there is only one connected cluster. We build the local matrices $A_{f}$ and use the software SIDNUR which implements the BDD method [35].

\subsection{Geometry and boundary conditions}

The position of the fractures is taken as uniform in the domain. Their orientation is uniform and their length follows a power law distribution of exponent 2.7 [8]. We take $p^{D}=1 \mathrm{~m}$ on the cube face at $y=L / 2$ and $p^{D}=20 \mathrm{~m}$ on the cube face at $y=-L / 2$. The transmissivity tensor is homogeneous and equal to $\mathscr{T}=T I d$, with $T=8.2 e-7 \mathrm{~m}^{2} \cdot \mathrm{s}^{-1}$. We consider 3 networks:

- L6 NF28: $L=6$ and $N_{f}=28$;

- L10 NF 18: $L=10$ and $N_{f}=18$;

- L10 NF24: $L=10$ and $N_{f}=24$.

\subsection{Mesh procedure and basic optimization}

The nonconforming mesh is generated according to the mesh procedure described in subsection 3.1. With this approach, adaptative mesh refinement can be done at the fracture level $[2,3,39,6]$.

A basic mesh coarsening consists in meshing finely only the fractures that take part significantly in the flow. Let us run a first simulation with a coarse mesh step $2 * \Delta$. The output flux for each fracture is computed, as well as the total output flux on the output cubic face. We choose to refine, with a mesh step $\Delta$, the fractures that have an output flux above $5 \%$ of the total output flux. The simulation is performed again on this refined mesh.

In table 1, we compare the mesh obtained with this basic mesh coarsening, socalled coarser mesh, with a mesh where the step is $\Delta$ for all fractures, so-called fine mesh. The min and mean of the quality mesh criterion $Q_{K} \in[0 ; 1]$ is also given, where $Q_{K}$ is defined for each triangle $K$ as [23]:

$$
Q_{K}=4 \sqrt{3} \frac{S_{K}}{h_{s}^{2}},
$$

with $S_{K}$ the surface of the triangle $K$ and $h_{s}=\sqrt{\sum_{i=1}^{3} h_{i}^{2}}$, with $h_{i}$ the length of the edge $i$ of the triangle $K$. The closer $Q_{K}$ is to 1 , the better the triangle quality is. 
Table 1 Comparison between a mesh with step $\Delta$ for all fractures and a mesh with step $\Delta$ for fractures with an output flux above $5 \%$ of the total output flux and $2 * \Delta$ otherwise

\begin{tabular}{|l|c|c|c|c|c|c|c|}
\hline Simulation name & $\Delta$ & \multicolumn{2}{|c|}{ Fine mesh - step $\Delta$} & \multicolumn{2}{c|}{ Coarser mesh - step $\Delta$ or $2 \Delta$} \\
\cline { 2 - 8 } & & Number of edges & $\operatorname{Min}\left(Q_{K}\right)$ & $\operatorname{Mean}\left(Q_{K}\right)$ & Number of edges & $\operatorname{Min}\left(Q_{K}\right)$ & $\operatorname{Mean}\left(Q_{K}\right)$ \\
\hline$L 6 \_N F 28$ & 0.05 & 122306 & 0.43 & 0.95 & 90533 & 0.23 & 0.95 \\
\hline$L 10 \_N F 18$ & 0.1 & 62409 & 0.45 & 0.95 & 57462 & 0.19 & 0.95 \\
\hline$L 10 \_N F 24$ & 0.1 & 78652 & 0.51 & 0.95 & 67765 & 0.25 & 0.95 \\
\hline
\end{tabular}

Table 1 shows that this basic mesh coarsening reduces the number of edges from $7.93 \%$ to $25.96 \%$ at the price of somehow lower mesh quality. Indeed the length of some fractures is too small compared with $2 \Delta$, yielding too few discretization points. As future work, we could define a minimal mesh step per fracture according to its length.

\subsection{Solution with SIDNUR}

Using the coarser mesh, we solve the linear system (12) with the BDD method. We checked these networks satisfy hypotheses $(H 1)-(H 2)$. From the computed values of $\Lambda_{m}$, we derive the unknowns $\Lambda_{s}$ and $\Lambda_{\Sigma}$ according to (8) - (9). The inner unknowns $\Lambda_{\text {in }}$ are derived locally in each fracture plane by solving small linear systems (see (10)). From these traces of hydraulic head unknowns, one can derive the mean head values and the fluxes [34]. Figures 2, 3 and 4 give the mean head values on the three DFN. Figure 5 displays the mean head values for the DFN L10_NF24 obtained by solving the linear system (12) with CHOLMOD to illustrate the good agreement of the results obtained with the two methods.

Table 2 gives the numbers $N_{i n}, N_{m}$ and $N_{s}$ with $N_{\Sigma}=N_{m}+N_{s}$ (hypothesis (H2)). This table also provides the number of PCG iterations, the final $L^{2}$-norm of the residual and the $L^{2}$-norm of the relative difference between the solutions $\left(\begin{array}{c}\Lambda_{i n} \\ \Lambda_{m}\end{array}\right)$ computed with SIDNUR and with the direct solver CHOLMOD [11].

Table 2 Solution with SIDNUR. Comparison with CHOLMOD

\begin{tabular}{|l|c|c|c|c|c|c|}
\hline Simulation name & $N_{i n}$ & $N_{m}$ & $N_{s}$ & \# PCG it. & PCG final residual & Comparison with CHOLMOD \\
\hline$L 6 \_N F 28$ & 89732 & 365 & 436 & 13 & $6.02 \mathrm{e}-17$ & $4.15 \mathrm{e}-12$ \\
\hline$L 10 \_N F 18$ & 56939 & 247 & 276 & 15 & $2.47 \mathrm{e}-18$ & $9.56 \mathrm{e}-13$ \\
\hline$L 10 \_N F 24$ & 66899 & 412 & 454 & 18 & $8.71 \mathrm{e}-19$ & $1.47 \mathrm{e}-12$ \\
\hline
\end{tabular}

On such small linear systems with very small CPU times, the solver SIDNUR is not competitive with respect to a direct solver. However this preliminary test phase demonstrates the possibility of solving linear system arising from a nonconforming discretization of networks satisfying hypotheses $(H 1)-(H 2)$ with the BDD 


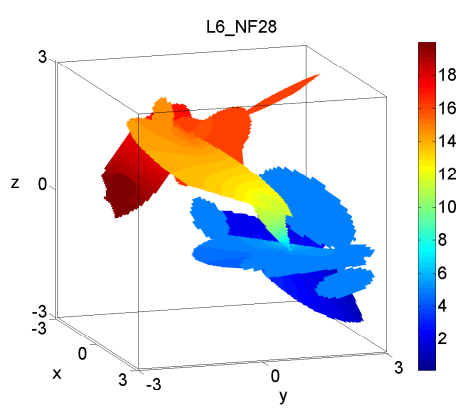

Fig. 2 L6_NF28 - Mean head - SIDNUR

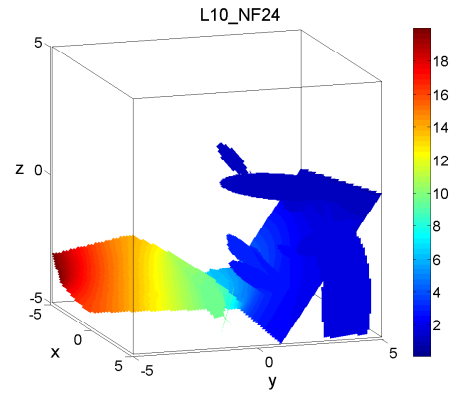

Fig. 4 L10 NF24 - Mean head - SIDNUR

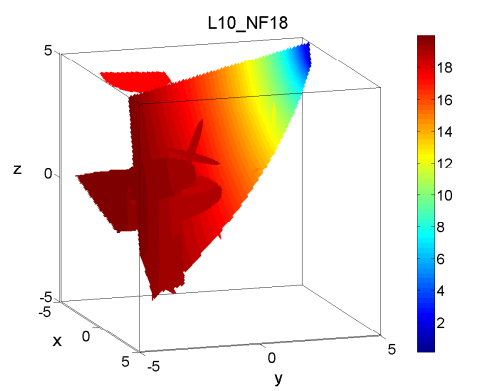

Fig. $3 L 10 \_N F 18$ - Mean head - SIDNUR

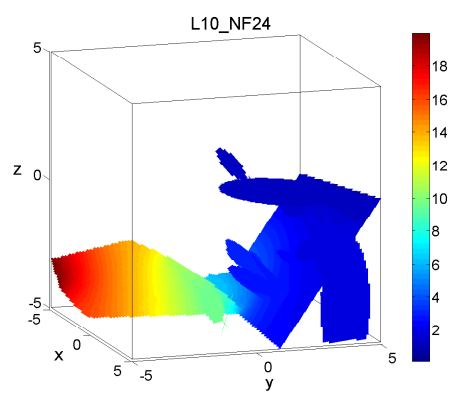

Fig. 5 L10_NF24 - Mean head - CHOLMOD

method. Using SIDNUR relies on a suitable decomposition of the local matrices. Moreover SIDNUR requires less RAM memory than a direct solver and is parallel.

\section{Conclusion}

This paper describes a Balancing Domain Decomposition method, implemented in the so-called SIDNUR solver, to simulate flow in DFN with a nonconforming mesh. DFN and local matrices are generated with the so-called MP_FRAC software. Our current work is to extend the method to more general discretizations, which do not satisfy hypotheses $(H 1)-(H 2)$, in the perspective of solving linear systems with several millions of unknowns. The parallelism of SIDNUR will be very helpful to reduce the time and memory costs. Moreover the very basic technic we use to coarsen the mesh could be improved by defining suitable a posteriori estimators.

Acknowledgements This work was supported by the French National Research Agency, with the ANR-07-CIS7 project MICAS, and by INRIA with the ARC-INRIA GEOFRAC project. 


\section{References}

1. Arbogast, T., Cowsar, L.C., Wheeler, M.F., Yotov, I.: Mixed finite element methods on nonmatching multiblock grids. SIAM J. Numer. Anal 37, 1295-1315 (2000)

2. Arnold, D.N., Brezzi, F.: Mixed and nonconforming finite element methods : implementation, postprocessing and error estimates. ESAIM: Mathematical Modelling and Numerical Analysis - Modlisation Mathmatique et Analyse Numrique 19(1), 7-32 (1985). URL http://eudml.org/doc/193443

3. Bernardi, C., Hecht, F.: Error indicators for the mortar finite element discretization of the Laplace equation. Mathematics of Computation 71(240), 1371-1403 (2002)

4. Bernardi, C., Maday, Y., Patera, A.T.: Domain decomposition by the mortar element method. In: Asymptotic and numerical methods for partial differential equations with critical parameters (Beaune, 1992), NATO Adv. Sci. Inst. Ser. C Math. Phys. Sci., vol. 384, pp. 269-286. Kluwer Acad. Publ., Dordrecht (1993)

5. Bernardi, C., Maday, Y., Patera, A.T.: A new nonconforming approach to domain decomposition: the mortar element method. In: Nonlinear partial differential equations and their applications. Collège de France Seminar, Vol. XI (Paris, 1989-1991), Pitman Res. Notes Math. Ser., vol. 299, pp. 13-51. Longman Sci. Tech., Harlow (1994)

6. Bernardi, C., Rebollo, T.C., Hecht, F., Mghazli, Z.: Mortar finite element discretization of a model coupling darcy and stokes equations. ESAIM: Mathematical Modelling and Numerical Analysis 42(3), 375-410 (2008). URL http: / / eudml . org/doc/250402

7. Bjørstad, P.E., Widlund, O.B.: Solving elliptic problems on regions partitioned into substructures. In: G. Birkhoff, A. Schoenstadt (eds.) Elliptic Problem Solvers II, pp. 245-256. Academic Press, New York (1984)

8. Bonnet, E., Bour, O., Odling, N., Davy, P., Main, I., Cowie, P., Berkowitz, B.: Scaling of fracture systems in geological media. Reviews of Geophysics 39(3), 347-383 (2001)

9. Bourgat, J.F., Glowinski, R., Le Tallec, P., Vidrascu, M.: Variational formulation and algorithm for trace operator in domain decomposition calculations. In: T. Chan, R. Glowinski, J. Périaux, O. Widlund (eds.) Domain Decomposition Methods, pp. 3-16. SIAM, Philadelphia, PA (1989)

10. Cacas, M.C., Ledoux, E., de Marsily, G., Barbeau, A., Calmels, P., Gaillard, B., Magritta, R.: Modeling fracture flow with a stochastic discrete fracture network: calibration and validation. 1. the flow model. Water Resources Research 26(3), 479-489 (1990)

11. Chen, Y., Davis, T.A., Hager, W.W., Rajamanickam, S.: Algorithm 887: Cholmod, supernodal sparse cholesky factorization and update/downdate. ACM Trans. Math. Softw. 35(3), 22:122:14 (2008). DOI 10.1145/1391989.1391995. URL http://doi.acm.org/10.1145/ 1391989.1391995

12. Cowsar, L.C., Mandel, J., Wheeler, M.F.: Balancing domain decomposition for mixed finite elements. Mathematics of computation 64(211), 989-1015 (1995). DOI $\{10.2307 / 2153480\}$

13. Davy, P., Bour, O., de Dreuzy, J.R., Darcel, C.: Flow in multiscale fractal fracture networks. In: 261 (ed.) Geological society, London, special publications, pp. 31-45 (2006)

14. Davy, P., Le Goc, R., Darcel, C., Bour, O., de Dreuzy, J.R., Munier, R.: A likely universal model of fracture scaling and its consequence for crustal hydromechanics. Journal of Geophysical Research 115, B10,411 (2010)

15. Dershowitz, W.S., Einstein, H.H.: Characterizing rock joint geometry with joint system models. Rock Mechanics and Rock Engineering 21(1), 2151 (1988)

16. Dohrmann, C.R.: A preconditioner for substructuring based on constrained energy minimization. SIAM J. Sci. Comput. 25, 246-258 (2003)

17. de Dreuzy, J.R., Pichot, G., Poirriez, B., Erhel, J.: Synthetic benchmark for modeling flow in 3D fractured media. Computers \& Geosciences 50, 59-71 (2013). DOI 10.1016/j.cageo.2012. 07.025. URL http://hal.inria.fr/hal-00735675

18. Dryja, M., Proskurowski, W.: On preconditioners for mortar discretization of elliptic problems. Numerical Linear Algebra with Applications 10(1-2), 65-82 (2003). DOI 10.1002/nla. 312. URL http://dx.doi.org/10.1002/nla.312 
19. Dverstop, B., Andersson, J.: Application of the discrete fracture network concept with field data: possibilities of model calibration and validation. Water Resources Research 25(3), 540550 (1989)

20. Erhel, J., de Dreuzy, J.R., Poirriez, B.: Flow simulations in three-dimensional discrete fracture networks. SIAM Journal on Scientific Computing 31(4), 2688-2705 (2009). DOI DOI:10. $1137 / 080729244$

21. Erhel, J., Guyomarc'h, F.: An augmented conjugate gradient method for solving consecutive symmetric positive definite systems. SIAM Journal on Matrix Analysis and Applications 21(4), 1279-1299 (2000)

22. Frank, J., Vuik, C.: On the construction of deflation-based preconditioners. SIAM J. Sci. Comput. 23(2), 442-462 (2001). DOI 10.1137/S1064827500373231. URL http://dx. doi.org/10.1137/S1064827500373231

23. Frey, P.J., George, P.L.: Maillages : applications aux éléments finis. Hermès sciences publ. DL1999 (53-Mayenne), Paris. URL http://opac. inria.fr/record=b1 094298

24. Gander, M.J., Tu, X.: On the origins of iterative substructuring methods. In: Domain Decomposition Methods (2013)

25. Glowinski, R., Wheeler, M.F.: Domain decomposition and mixed finite element methods for elliptic problems. In: R. Glowinski, G.H. Golub, G.A. Meurant, J.P. Eds (eds.) Domain Decomposition Methods for Partial Differential Equations, pp. 144-172. SIAM, Philadelphia (1988)

26. Kim, H.H., Dryja, M., Widlund, O.B.: A bddc method for mortar discretizations using a transformation of basis. SIAM J. Numerical Analysis 47(1), 136-157 (2008)

27. Le Tallec, P.: Domain decomposition methods in computational mechanics. In: J.T. Oden (ed.) Computational Mechanics Advances, vol. 1 (2), pp. 121-220. North-Holland (1994)

28. Le Tallec, P., De Roeck, Y., Vidrascu, M.: Domain decomposition methods for large linearly elliptic three-dimensional problems. Journal of Computational and Applied Mathematics 34, 93-117 (1991)

29. Mandel, J.: Balancing domain decomposition. Communications in Applied Numerical Methods 9, 233241 (1993)

30. Mandel, J., Brezina, M.: Balancing domain decomposition: Theory and computations in two and three dimensions. Tech. Rep. UCD/CCM 2, Center for Computational Mathematics, University of Colorado at Denver (1993)

31. Meurant, G.: Computer Solution of Large Linear Systems. Elsevier Science B.V. (1999)

32. Pencheva, G., Yotov, I.: Balancing domain decomposition for mortar mixed finite element methods. Numerical Linear Algebra with Applications 10(1-2), 159-180 (2003). DOI 10. 1002/nla.316. URL http://dx.doi.org/10.1002/nla.316

33. Pichot, G., Erhel, J., de Dreuzy, J.R.: A mixed hybrid mortar method for solving flow in discrete fracture networks. Applicable Analysis 89(10), 1629-1643 (2010)

34. Pichot, G., Erhel, J., de Dreuzy, J.R.: A generalized mixed hybrid mortar method for solving flow in stochastic discrete fracture networks. SIAM Journal on Scientific Computing 34(1), B86B105. (20 pages) (2012)

35. Poirriez, B.: Etude et mise en oeuvre d'une méthode de sous-domaines pour la modélisation de l'écoulement dans des réseaux de fractures en 3d. Ph.D. thesis, University of Rennes 1 (2011)

36. Quarteroni, A., Valli, A.: Domain Decomposition Methods for Partial Differential Equations. Oxford Science Publications (1999)

37. Tang, J.M., Nabben, R., Vuik, C., Erlangga, Y.A.: Comparison of two-level preconditioners derived from deflation, domain decomposition and multigrid methods. J. Sci. Comput. 39(3), 340-370 (2009). DOI 10.1007/s10915-009-9272-6. URL http://dx.doi.org/10 . 1007 /s10915-009-9272-6

38. Vohralik, M., Maryska, J., Severyn, O.: Mixed and nonconforming finite element methods on a system of polygons. Applied Numerical Mathematics 57, 176-193 (2007)

39. Wheeler, M.F., Yotov, I.: A posteriori error estimates for the mortar mixed finite element method. SIAM J. Numer. Anal. 43(3), 1021-1042 (2005). DOI 10.1137/S0036142903431687. URL http: / /dx.doi.org/10.1137/s0036142903431687 